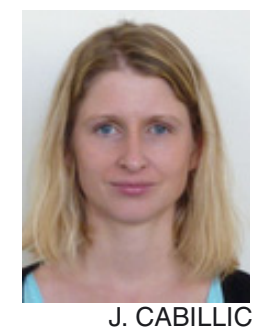

\title{
Développement et validation d'une méthode de référence pour l'analyse des HAP dans l'eau totale dans le contexte de la DCE
}

\section{Development and validation of a reference method for the analysis of PAHs in whole water in accordance to the WFD}

\author{
Julie CABILLIC ${ }^{1}$, Carine FALLOT ${ }^{1}$, Sebastian HEIN² ${ }^{2}$ Béatrice LALERE ${ }^{1}$ et Rosemarie PHILIPP ${ }^{2}$ \\ ${ }^{1}$ Laboratoire national de métrologie et d'essais (LNE), 1 rue Gaston Boissier, 75724 Paris Cedex 15, France, julie.cabillic@lne.fr. \\ ${ }^{2}$ Bundesanstalt für Materialforschung und-prüfung (BAM), Richard-Willstaetter-Str. 11, 12489 Berlin, Allemagne.
}

\section{Résumé}

Afin de garantir la qualité des eaux en Europe, la Commission Européenne a demandé aux États Membres, de leur fournir des résultats de mesure comparables et traçables pour l'analyse des polluants critiques. Devant ces enjeux considérables pour la protection de l'environnement, l'apport de la métrologie est nécessaire pour améliorer la fiabilité des analyses et permettre la comparabilité des résultats. C'est pourquoi, le projet de recherche européen Euramet/EMRP/ENV08 «Traçabilité des mesures pour la surveillance des polluants critiques de la DCE, Directive Cadre européenne sur l'Eau (2000/60/CE) », financé par l'EMRP, avait pour objectif de fournir des éléments pour la mise en place d'une base métrologique. Des méthodes de référence primaires permettant d'analyser des substances dangereuses prioritaires y sont développées pour répondre aux exigences de la DCE en termes de niveaux de performance (limites de quantification et incertitudes). Les hydrocarbures aromatiques polycycliques (HAP), substances dangereuses prioritaires « orphelines » de méthodes DCE compatibles, font partie des polluants ciblés par le projet. Le développement et la validation de la méthode d'analyse des HAP dans l'eau totale ont été réalisés. La méthode qui satisfait au mieux les exigences de la DCE en termes de concentrations et d'incertitudes accessibles est l'extraction en phase solide (SPE) sur un support disque suivie d'une analyse par dilution isotopique associée à la chromatographie en phase gazeuse couplée à la spectroscopie de masse (DI-CG/SM).

MOTS CLÉS : HAP, MÉTHODE DE RÉFÉRENCE, LIMITE DE QUANTIFICATION, INCERTITUDE, SPE DISQUE.

\footnotetext{
Abstract

To ensure the water quality in Europe, the European Commission asked the member states to provide comparable and traceable
}

measurement results for the analysis of critical pollutants. Considering the increasing environmental challenges, the contribution of metrology is necessary both for the improvement of analysis reliability and the comparability of results. For this reason the European research project Euramet/EMRP/ENV08 "Traceable measurements for monitoring critical pollutants under the European Water Framework Directive (WFD-2000/60/EC)", funded by the EMRP, aimed to provide the elements for the development of a metrological basis. The project develops primary reference methods enabling the analysis of priority hazardous substances in order to meet the WFD requirements in terms of performance levels (limit of quantification and uncertainties). Polycyclic aromatic hydrocarbons (PAHs) - priority hazardous substances for which no DCE compatible methods exist-are among the three families of pollutants targeted by the project. This paper tackles the development and validation of the PAH analysis method on whole water samples. The method that best meets the requirements of the WFD in terms of level of concentration and uncertainty is the solid phase extraction (SPE) method with SPE-disks, followed by isotope dilution analysis combined with gas chromatography mass spectrometry (ID-GC/MS).

KEY WORDS: PAH, REFERENCE METHODS, LIMIT OF QUANTIFICATION, UNCERTAINTY, SPE DISK.

\section{Contexte et objectifs de l'étude}

En Europe, les ressources et les milieux naturels se dégradent, impactés par les activités industrielles, urbaines et agricoles. Le contrôle de la pollution de l'eau est devenu un enjeu prioritaire pour ses conséquences sur la santé humaine et sur l'environnement. La qualité de l'eau et des milieux aquatiques a toujours été une 
préoccupation majeure de la politique de l'Union européenne. C'est pourquoi la DCE, directive européenne cadre sur l'eau (2000/60/CE) [1] a été établie le 23 octobre 2000 afin d'obliger les pays de la Communauté européenne à restaurer et protéger la qualité de leurs ressources souterraines et superficielles en eau.

Selon la DCE, un «bon état chimique» pour un plan d'eau est obtenu lorsque les concentrations des substances prioritaires dans l'eau ou le biote sont en dessous des normes de qualité environnementale $(N Q E)$ fixées par la directive 2008/105/CE [2]. En complément, la directive QA/QC 2009/90/CE [3], directive fille de la DCE, établit des spécifications techniques pour l'analyse chimique et la surveillance de l'état des eaux, afin de garantir des résultats de mesure justes et comparables aux $N Q E$ pour les polluants prioritaires de la DCE, dans les eaux totales (eaux et matières en suspension). Ainsi, les méthodes analytiques mises en œuvre pour cette surveillance doivent satisfaire aux exigences en termes de limites de quantification $(L Q \leq 30 \%$ des $N Q E)$ et incertitudes $(U(k=2) \leq 50 \%$ à la $N Q E)$.

Par ailleurs, afin de permettre le contrôle qualité, la Directive précise que « Les laboratoires doivent apporter la preuve de leurs compétences dans l'analyse des mesurandes physicochimiques ou chimiques : par leur participation à des programmes d'essais d'aptitude couvrant les méthodes d'analyse (...), par l'analyse de matériaux de référence disponibles, représentatifs des échantillons prélevés et contenant des niveaux de concentration appropriés au regard des normes de qualité environnementale applicables (...)» (Article 6, point 2 [3]). Or, à ce jour, s'il existe bien des comparaisons inter-laboratoires (CIL) pour ces composés, les niveaux de concentrations ne sont pas compatibles avec les exigences de la DCE et la valeur de référence est une valeur consensuelle qui n'est pas traçable au Système international d'unités (SI). Il y a de ce fait un fort besoin de pouvoir disposer de valeurs de référence établies à partir de méthodes de référence primaires ${ }^{1}$.

C'est pour fournir ces outils métrologiques que le projet de recherche européen ENV08 «Traçabilité des mesures pour la surveillance des polluants critiques de la Directive Cadre Européenne sur l'Eau (2000/60/CE)» financé par l'EMRP (European Metrology Research Programme), a été lancé en 2011 pour une durée de 3 ans. C'est un projet pluridisciplinaire coordonné par le BAM, Bundesanstalt für Materialforschung und prüfung (Allemagne), impliquant 14 établissements (instituts nationaux de métrologie, instituts désignés, le Joint Research Center et instituts de recherche d'excellence) de 10 pays différents. Un des objectifs était de mettre à

\footnotetext{
1 «Une méthode de référence primaire est une méthode ayant les plus hautes qualités métrologiques dont toutes les étapes sont décrites et comprises, pour laquelle un bilan complet d'incertitudes peut être établi en référence aux unités du S.I. et dont les résultats ne font pas référence à la mesure de la quantité de matière d'un étalon de même nature du mesurande pour mesurer un analyte dans un échantillon », BIPM, Comité Consultatif pour la Quantité de Matière, 1995, 1, 38 p.
}

Tableau 1

Liste des HAP étudiés.

\begin{tabular}{|l|c|c|}
\hline \multicolumn{1}{|c|}{ Composé } & $\begin{array}{c}\boldsymbol{N Q E} \\
\left(\boldsymbol{\mu g} \cdot \mathbf{L}^{-\mathbf{1}}\right)\end{array}$ & $\begin{array}{c}\mathbf{L Q}= \\
\mathbf{3 0} \% \mathbf{N Q E} \\
\left(\boldsymbol{\mu g} \cdot \mathbf{L}^{-1}\right)\end{array}$ \\
\hline Anthracène (Ant) & 0,1 & 0,03 \\
\hline Fluoranthène (Fluo) & 0,1 & 0,03 \\
\hline Benzo(b)fluoranthène (BbF) & 0,03 & 0,009 \\
\hline Benzo(k)fluoranthène (BkF) & & \\
\hline Benzo(a)pyrène (BaP) & 0,05 & 0,015 \\
\cline { 1 - 1 } Benzo(g,h,i)pérylène (BghiP) & \multirow{2}{*n}{0,002} & 0,0006 \\
\cline { 1 - 1 } Indéno(1,2,3-cd)pyrène (IndenoP) & &
\end{tabular}

disposition de la communauté scientifique des méthodes de référence primaires répondant aux performances exigées par la DCE.

À ce jour, de telles méthodes ne sont pas disponibles pour tous les polluants de la liste des 33 substances prioritaires [4,5] : les méthodes européennes issues des groupes de normalisation CEN/TC230 «Analyse de l'eau » et ISO/TC147 «Qualité de l'eau » ne sont pas toutes compatibles avec la DCE en termes de performance ( $L Q$ et incertitudes) et d'applicabilité à l'eau totale. Un inventaire réalisé par le CEN/TC230 (Water Analysis) en 2004 avait identifié plus particulièrement un manque de méthodes suffisamment performantes pour les hydrocarbures aromatiques polycycliques (HAP) [6-8].

Le LNE (Laboratoire national de métrologie et d'essais, France) a coordonné le développement et la validation d'une méthode de référence primaire pour le dosage de HAP dans l'eau totale mettant en œuvre la dilution isotopique $^{2}$ (DI). Quatre instituts ont été impliqués dans cette étude : le BAM, le BRML (Biroul Roman de Metrologie Legala, Roumanie), le TUBITAK UME (Ulusal Metroloji Enstitusu, Turquie).

Le premier objectif de cette étude était de respecter les exigences de la DCE en termes de performances $(L Q$ et incertitude). En effet, ces paramètres représentent une difficulté analytique pour certains HAP (tab. 1) puisqu'ils sont à des niveaux très bas de concentration (plus particulièrement pour la somme des benzo(b)fluoranthène et benzo(k)fluoranthène et des benzo $(\mathrm{g}, \mathrm{h}, \mathrm{i})$ pérylène et indéno(1,2,3-cd)pyrène).

Enfin, la méthode doit être applicable sur des échantillons d'eau totale. La difficulté consiste à doser les HAP présents dans la phase dissoute et particulaire en une seule étape. Il est connu d'après la bibliographie $[4,5,9]$

\footnotetext{
${ }^{2}$ Le principe de la dilution isotopique consiste à ajouter aux composés recherchés, des molécules au sein desquelles des atomes sont remplacés par des isotopes stables comme, par exemple, la substitution d'un atome d'hydrogène ${ }^{1} \mathrm{H}$ par un atome de deutérium ${ }^{2} \mathrm{D}$ ou d'un atome de carbone ${ }^{12} \mathrm{C}$ par un atome de carbone ${ }^{13} \mathrm{C}$. La mesure du rapport entre l'intensité du signal de la molécule et celle de la molécule marquée permet de déterminer directement le rapport de quantité de matière entre les deux molécules et donc de déduire la concentration du composé.
} 
que l'extraction d'échantillons aqueux riches en matières en suspension (MES) pose problème en raison de phénomènes de colmatage et de rendements incomplets pouvant conduire à une sous-estimation du résultat final. C'est pourquoi deux approches de l'étape d'extraction ont été comparées :

- deux extractions distinctes après séparation des particules en suspension et de l'échantillon filtré (approche 1); cette approche permettra d'extraire les particules en suspension avec une méthode d'extraction solide (extraction accélérée par solvant à chaud) développée au cours du projet ;

- une seule analyse de l'eau totale (approche 2) [10,11].

\section{Matériels et méthodes}

\subsection{Produits chimiques et réactifs}

Les solutions étalons ont été préparées gravimétriquement à partir d'étalons individuels en poudre de grande pureté (>99\%). La sélection des étalons marqués pour la mise en œuvre de la dilution isotopique est un point critique. Pour l'analyse des matrices solides (particules et sédiments), des travaux bibliographiques ont montré que le choix de l'étalon marqué pouvait avoir un impact sur le résultat final du fait de la différence de comportements avec le composé natif $[11,12]$. En effet, lors d'une précédente étude sur les HAP dans les particules [13], le LNE avait comparé les rendements d'extraction par solvant pressurisé à chaud (PLE) obtenus pour un HAP natif et les composés marqués au ${ }^{13} \mathrm{C}$ et au ${ }^{2} \mathrm{D}$ correspondants. Les résultats avaient permis de mettre en évidence que le marquage au ${ }^{13} \mathrm{C}$ conduisait à de meilleurs résultats en termes de rendements qu' avec le marquage au ${ }^{2} \mathrm{D}$. Ce phénomène peut s'expliquer par des échanges rapides $\mathrm{H} /{ }^{2} \mathrm{D}$ qui se produisent au cours des différentes étapes du processus d'analyse des HAP et donc entrainent une perte des $\mathrm{HAP}^{2} \mathrm{D}$. Chaque étalon marqué a été analysé séparément pour vérifier qu'il ne contenait pas de composé non marqué. La bibliographie indique également que les $\mathrm{HAP}^{2} \mathrm{D}$ pouvaient avoir une plus grande affinité pour les sites actifs du «liner» (ou insert) dans le système d'injection du CG se traduisant par une adsorption privilégié sur les parois [14]. Des comportements différents entre les HAP et $\mathrm{HAP}^{2} \mathrm{D}$ ont été également observés dans la chambre d'ionisation du spectromètre de masse [15].

Une solution d'acide humique a été préparée afin de doper artificiellement une eau minérale aux niveaux de concentration couramment retrouvés dans les eaux de surface (de l'ordre de $5 \mathrm{mg} \cdot \mathrm{L}^{-1}$ de carbone organique dissous) : 7,50 g d'acide humique synthétique (Sigma Aldrich, H16752-100g) ont été ajouté gravimétriquement à $500 \mathrm{~mL}$ d'eau ultra pure. La solution a été passée aux ultrasons pendant $1 \mathrm{~h}$ à $40^{\circ} \mathrm{C}$ puis centrifugée à 9000 tours par minute pendant 20 min et filtrée à $0,45 \mu \mathrm{m}$. La solution d'acide humique préparée contient $2 \mathrm{~g} \cdot \mathrm{L}^{-1}$ de carbone organique dissous.

Deux types de sédiments fournis par l'IRMM ont été utilisés pour doper une eau minérale et simuler la
Tableau 2

Concentrations de HAP dans les sédiments utilisés pour le dopage des eaux synthétiques.

\begin{tabular}{|c|c|c|}
\hline \multirow{2}{*}{ HAP } & $\begin{array}{c}\text { MES blanc } \\
\text { IRMM }\end{array}$ & $\begin{array}{c}\text { MES } \\
\text { HAP IRMM }\end{array}$ \\
\cline { 2 - 3 } & \multicolumn{2}{|c|}{ Concentration $\left(\mathbf{n g} \cdot \mathbf{g}^{\mathbf{- 1}}\right)$} \\
\hline Anthracène & 7,4 & 593 \\
\hline Fluoranthène & 53,2 & 9477 \\
\hline Benzo(b)fluoranthène & 44,1 & 2976 \\
\hline Benzo(k)fluoranthène & 22,3 & 1700 \\
\hline Benzo(a)pyrène & 38,9 & 2121 \\
\hline Indéno(1,2,3-c,d)pyrène & 43,1 & 2725 \\
\hline Benzo(g,h,i)pérylène & 47,0 & 2934 \\
\hline
\end{tabular}

présence de matières en suspension. Ce sont des sédiments contenant une quantité connue de HAP et dont la taille de particules étaient comprises entre $9 \mu \mathrm{m}$ et $12,5 \mu \mathrm{m}$ (ce qui correspond à la taille moyenne de MES retrouvées dans les eaux de surface). Les concentrations en HAP pour chaque sédiment sont fournies dans le tableau 2.

\subsection{Préparation des échantillons}

\subsection{1. État de l'art}

Comme il n'existe pas de matériau de référence de HAP dans l'eau totale, il a été nécessaire de créer des matrices synthétiques, représentatives des eaux naturelles, pour évaluer les performances des méthodes testées. L'utilisation d'une même eau synthétique représentative des eaux naturelles, par les différents partenaires impliqués dans l'étude, s'est imposée pour assurer la comparabilité des méthodes d'analyse développées.

Pour définir un protocole de préparation de cette eau synthétique, la composition des eaux de surface a été étudiée. Ces eaux contiennent différents éléments chimiques dissous, du fait de leur cheminement dans le sol et du contact avec l'atmosphère. $25 \%$ à $50 \%$ du carbone organique dissous (COD) des eaux de surface provient notamment des substances humiques [12]. Ces substances peuvent avoir un effet sur l'analyse des HAP dans l'eau $[16,17]$ en réduisant l'efficacité de l'étape d'extraction ou en apportant de possibles interférences lors de l'analyse.

La DCE imposant l'analyse de l'eau totale, il a donc été indispensable d'introduire des matières en suspension pour respecter ce critère.

\subsubsection{Méthode de préparation des échantillons synthétiques}

Un protocole commun de préparation d'une eau synthétique a donc été défini :

- 1 L d'eau minérale (Evian) est introduit gravimétriquement dans un flacon ambré d'un litre, 
- différents niveaux d'acides humiques compris entre 0 et $10 \mathrm{mg} \cdot \mathrm{L}^{-1}$ et/ou différentes quantités de sédiments (afin de simuler divers types d'eau) sont ajoutés gravimétriquement,

- pour mettre en œuvre la dilution isotopique, une quantité de composé marqué proche de celle du composé contenu dans l'échantillon à analyser est introduite gravimétriquement.

L'échantillon est agité pour homogénéisation puis mis à l'équilibre avant extraction. Les échantillons d'eau ont été dopés en HAP et HAP marqués 24 heures avant analyse pour garantir un équilibre optimum avec les acides humiques selon les recommandations de Bercaru et al. [18] et Van de Kreeke et al. [19].

\subsection{Méthode chromatographique couplée à la spectrométrie de masse}

La méthode de mesure primaire pour le dosage des HAP repose exclusivement sur la dilution isotopique associée à la chromatographie gazeuse couplée à la spectrométrie de masse (DI-CG/SM). L'analyse est réalisée avec un chromatographe en phase gazeuse PerkinElmer équipé d'une colonne capillaire apolaire de type Agilent DB EUPAH $(60 \mathrm{~m} \times 250 \mu \mathrm{m} \times 0,25 \mu \mathrm{m})$. La programmation de température a été optimisée pour permettre une séparation des différents HAP et plus particulièrement des co-élutions critiques telles que l'anthracène et le phénanthrène $(\mathrm{m} / \mathrm{z} 178)$, les benzo(b)fluoranthène, benzo $(\mathrm{k})$ fluoranthène et benzo(j)fluoranthène $(\mathrm{m} / \mathrm{z}, 252)$, le benzo(a)pyrène et le benzo(e)pyrène $(\mathrm{m} / \mathrm{z} 252)$ ainsi que l'indéno(123-cd)pyrène, le dibenz(ah)anthracène et le dibenzo(a,c)anthracène $(m / z, 276,278)$.

Le programme optimisé du CG est le suivant : température initiale $50{ }^{\circ} \mathrm{C}$ (pendant $2,25 \mathrm{~min}$ ) jusqu'à $200{ }^{\circ} \mathrm{C}$ à $45^{\circ} \mathrm{C}$ par minute puis jusqu'à $300^{\circ} \mathrm{C}$ à $10^{\circ} \mathrm{C}$ par minute (pendant $25 \mathrm{~min}$ ). Le volume d'injection a été fixé à $5 \mu \mathrm{L}$ en mode «solvent vent »: température de l'injecteur fixée à $50{ }^{\circ} \mathrm{C}$ (pendant $1 \mathrm{~min}$ ) jusqu'à $300{ }^{\circ} \mathrm{C}$ à $200^{\circ} \mathrm{C}$ par minute. L'hélium est utilisé comme gaz vecteur à un débit de $1 \mathrm{~mL} \cdot \mathrm{min}^{-1}$. Les paramètres du $\mathrm{SM}$ sont les suivant :

- température de la ligne de transfert : $300{ }^{\circ} \mathrm{C}$;

- température de la source : $240{ }^{\circ} \mathrm{C}$;

- mode EI : $70 \mathrm{eV}$.

\subsection{Protocole de nettoyage de la verrerie}

Afin de limiter les contaminations croisées provenant du matériel utilisé lors des essais, un protocole de nettoyage spécifique de la verrerie a été mis en place. Il comprend une étape de nettoyage aux détergents et rinçage à l'eau distillée suivie d'une calcination à $450{ }^{\circ} \mathrm{C}$ pendant $5 \mathrm{~h}[20,21]$
Tableau 3

Paramètres d'extraction par PLE.

\begin{tabular}{|c|c|}
\hline Solvant & Acétone/hexane $(2: 1)$ \\
\hline Température & $120{ }^{\circ} \mathrm{C}$ \\
\hline Pression & $140 \mathrm{bar}(14 \mathrm{MPa})$ \\
\hline Temps statique & $10 \mathrm{~min}$ \\
\hline Temps de chauffage & $6 \mathrm{~min}$ \\
\hline Flush & $70 \%$ \\
\hline Temps de purge & $100 \mathrm{~s}$ \\
\hline Nombre de cycles & 2 \\
\hline
\end{tabular}

\subsection{Méthode d'extraction par solvant pressurisé à chaud}

L'extraction des matières en suspension est réalisée par solvant pressurisé à chaud (PLE). Les différents paramètres de cette méthode sont résumés dans le tableau 3.

\section{Développement et validation d'une méthode d'analyse des HAP dans l'eau totale}

\subsection{Extraction des HAP dans l'eau totale sur phase solide}

La préparation de l'échantillon est une étape clé du processus d'analyse. C'est une des principales sources d'incertitudes du résultat d'analyse influant particulièrement sur la limite de quantification, la fidélité et la répétabilité de l'analyse. L'analyse des HAP dans l'eau totale nécessite une étape de concentration et de passage dans un solvant adapté à une analyse par CG/SM. L'extraction sur phase solide (SPE) [22,23] est une technique très utilisées pour l'analyse des HAP dans l'eau. Elle a été optimisée sur des échantillons d'eau synthétiques contenant des acides humiques et des matières en suspension. Deux types de supports ont été testés : les cartouches et les disques.

\subsubsection{SPE sur supports cartouches}

Cinq types de cartouches, traditionnellement utilisées pour l'extraction des HAP dans l'eau [20,24,25], ont été testées sur des échantillons d'eau synthétique contenant des acides humiques (environ $1 \mathrm{mg} \cdot \mathrm{L}^{-1}$ ) et enrichis en HAP au niveau de la $N Q E$. Compte tenu de la présence d'acides humiques dans les échantillons et de leur impact potentiel sur l'efficacité de l'extraction (colmatage, fixation spécifique...), plusieurs supports de cartouches ont été testés : quatre phases solides : C18 et polymériques (connues pour ne pas retenir les interférents) ou combinaison de deux phases (les interférents liés aux acides humiques sont retenus sur une phase spécifique alors que les HAP sont élués). Différents volumes de phase solide ont été étudiés afin de retenir le maximum d'acides humiques sur la cartouche tout en évitant que le volume de phase trop élevé induise une élution incomplète des composés. Les résultats obtenus sont résumés dans le tableau 4.

Pour l'ensemble des cartouches, les rendements d'extraction sont de l'ordre de $78 \%$ à $91 \%$ pour les composés 
Tableau 4

Rendements d'extraction des HAP dans l'eau contenant des acides humiques obtenus pour différents types de cartouches $(n=2$, la dispersion moyenne des résultats est de $20 \%$ ).

\begin{tabular}{|c|c|c|c|c|c|c|c|}
\hline & \multicolumn{7}{|c|}{ Rendement (\%) } \\
\hline Type de cartouche & Ant & Fluo & $\mathrm{BbF}$ & $\mathrm{BkF}$ & $\mathrm{BaP}$ & Indeno & BghiP \\
\hline Oasis HLB $200 \mathrm{mg} / 6 \mathrm{~mL}$ (Waters) & 90 & 91 & 19 & 25 & 14 & 11 & 10 \\
\hline Oasis HLB $1 \mathrm{~g} / 20 \mathrm{~mL}$ (Waters) & 81 & 78 & 29 & 28 & 26 & 15 & 14 \\
\hline Strata PAH 1,5 g/6 mL (Phenomenex) & 84 & 84 & 73 & 74 & 70 & 56 & 60 \\
\hline Chromabond NH2-C18 500 mg-1 g/6 mL (Macherey Nagel) & 87 & 90 & 67 & 64 & 64 & 46 & 51 \\
\hline Chromabond C18 PAH 2 g/6 mL (Macherey Nagel) & 81 & 85 & 70 & 69 & 69 & 55 & 60 \\
\hline
\end{tabular}

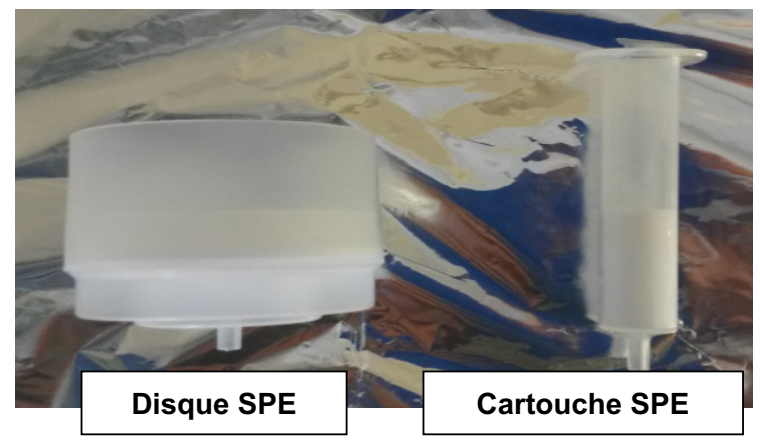

Fig. 1. - Présentation d'un disque SPE et d'une cartouche SPE.

légers : l'anthracène et le fluoranthène. Les rendements sont plus faibles pour les composés les plus lourds (du benzo(b)fluoranthène au benzo(ghi)pérylène) : de $10 \%$ à $65 \%$. Ce phénomène peut être expliqué par une compétition des macromolécules organiques provenant des acides humiques et des HAP à extraire sur les sites d'adsorption disponibles [25], en particulier pour les plus lourds (HAP dont le nombre de cycles est supérieur à 5).

Pour la cartouche OASIS HLB (phase polymérique), l'augmentation de la quantité de phase de $200 \mathrm{mg}$ à $1 \mathrm{~g}$ n'améliore pas de manière significative les rendements des composés lourds. Ce phénomène pourrait être expliqué par la non-spécificité de la phase pour les HAP qui donnerait lieu à une compétition entre les HAP et les acides humiques en faveur de ces derniers.

Les trois cartouches qui ont permis d'obtenir les rendements les plus élevés pour les composés lourds (de $46 \%$ à $74 \%$ ) sont les cartouches Strata PAH $1,5 \mathrm{~g} / 6 \mathrm{~mL}$ (Phenomenex), la Chromabond NH2-C18 $500 \mathrm{mg}-1 \mathrm{~g} / 6 \mathrm{~mL}$ (Macherey-Nagel) et la Chromabond C18 PAH 2 g/6 mL (Macherey-Nagel). Ce sont des cartouches contenant des quantités de phases équivalentes et qui possèdent toutes les trois des phases spécifiques à l'extraction des HAP.

Cependant ces cartouches ne permettaient pas d'atteindre des rendements suffisants pour les composés lourds. Par ailleurs, pour des échantillons chargés en MES et/ou en acides humiques, l'extraction avec les cartouches SPE n'était pas possible. En effet, la géométrie des supports d'extraction a un impact sur le processus d'extraction : plus la surface de contact est faible, plus les phénomènes de colmatage seront élevés (fig. 1).

\subsection{2. $\quad$ SPE sur supports disques}

La technologie laminaire des disques d'extraction permet une très grande capacité de filtrage et une plus grande capacité d'interaction et d'échange entre microparticules et analytes. Le nombre de phases disponibles est plus restreint pour ce type de support. Les deux types de phases les plus adaptés à l'analyse des HAP ont été testés sur des échantillons d'eau contenant $5 \mathrm{mg} \cdot \mathrm{L}^{-1}$ d'acides humiques :

- BAKERBOND Speedisk ${ }^{\mathrm{TM}}$ C18 (JT BAKER) [26] ;

- BAKERBOND Speedisk ${ }^{\mathrm{TM}} \mathrm{H}_{2} \mathrm{O}$ phobic DVB (JT BAKER) [21], phase polymérique.

Les résultats obtenus sont résumés dans le tableau 5.

Les rendements sont du même ordre de grandeur pour les deux types de disques (de $75 \%$ à $107 \%$ ).

Les performances des deux supports étant équivalentes, la BAKERBOND Speedisk ${ }^{\mathrm{TM}} \mathrm{C} 18$ de JT BAKER a été sélectionnée pour l'étape de validation pour des raisons de coûts et de disponibilités.

De manière générale, l'extraction avec les disques présente de nombreux avantages comparée à l'extraction sur cartouches :

- meilleurs rendements pour les composés les plus lourds (du benzo(b)fluoranthène au benzo(ghi)pérylène) : $75 \%$ à $103 \%$ pour les disques et $10 \%$ à $65 \%$ pour les cartouches ;

- temps d'extraction plus court dû à la plus grande surface d'échange des disques et à la diminution des risques de colmatage : $30 \mathrm{~min}$ à $40 \mathrm{~min}$ pour les disques contre $2 \mathrm{~h}$ à $3 \mathrm{~h}$ pour les cartouches.

\subsubsection{Optimisation de l'extraction SPE sur supports disques}

La méthode d'extraction SPE disque BAKERBOND Speedisk ${ }^{\mathrm{TM}}$ C18 de JT BAKER a été optimisée pour obtenir des rendements de l'ordre de $100 \%$.

Différents types et mélanges de solvants ont été testés pour optimiser l'élution des composés du disque (acétonitrile, acétonitrile/dichlorométhane, acétone/dichlorométhane, acétone/hexane). 
Tableau 5

Rendements d'extraction des HAP dans une eau contenant des acides humiques pour deux types de disques SPE $(n=3$, la dispersion moyenne des résultats est de $20 \%$ ).

\begin{tabular}{|l|c|c|c|c|c|c|c|}
\cline { 2 - 9 } \multicolumn{1}{c|}{} & \multicolumn{9}{c|}{ Rendement (\%) } \\
\cline { 2 - 9 } \multicolumn{1}{c|}{} & Ant & Fluo & BbF & BkF & BaP & Indeno & BghiP \\
\hline BAKERBOND Speedisk ${ }^{\mathrm{TM}}$ C18 (JT Baker) & 92 & 102 & 99 & 86 & 94 & 75 & 97 \\
\hline BAKERBOND Speedisk ${ }^{\mathrm{TM}}$ H2O phobic DVB (JT Baker) & 99 & 107 & 102 & 90 & 100 & 103 & 103 \\
\hline
\end{tabular}

Tableau 6

Protocole d'extraction SPE disque.

\begin{tabular}{|l|c|c|c|}
\hline \multicolumn{1}{|c|}{ Étape } & Solvant & $\begin{array}{c}\text { Temps de } \\
\text { trempage } \\
\text { (min) }\end{array}$ & $\begin{array}{c}\text { Temps de } \\
\text { séchage } \\
\text { (s) }\end{array}$ \\
\hline Conditionnement 1 & hexane & 1 & \\
\hline Conditionnement 2 & acétone & 1 & \\
\hline Conditionnement 3 & eau & 1 & \\
\hline \multicolumn{3}{|c|}{ Percolation de l'échantillon } \\
\hline \multicolumn{3}{|c|}{ Rinçage à l'eau ultra pure } \\
\hline \multicolumn{3}{|c|}{ Séchage pendant 5 min } \\
\hline Élution 1 & acétone & 2 & 20 \\
\hline Élution 2 & hexane & 2 & 40 \\
\hline Élution 3 & hexane & 1 & 20 \\
\hline Élution 4 & hexane & 1 & 20 \\
\hline Élution 5 & hexane & 1 & 20 \\
\hline Élution 6 & hexane & 1 & 40 \\
\hline
\end{tabular}

Tableau 7

Rendement d'extraction des HAP dans l'eau totale avec la méthode finalisée par SPE disque - DI - CG/SM $(n=3)$.

\begin{tabular}{|l|c|c|}
\hline HAP & Rendement & Écart type \\
\hline Anthracène & $72 \%$ & $17 \%$ \\
\hline Fluoranthène & $87 \%$ & $8 \%$ \\
\hline Benzo(b)fluoranthène & $95 \%$ & $6 \%$ \\
\hline Benzo(k)fluoranthène & $95 \%$ & $6 \%$ \\
\hline Benzo(a)pyrène & $98 \%$ & $11 \%$ \\
\hline Indeno(123-cd)pyrène & $91 \%$ & $9 \%$ \\
\hline Benzo(ghi)pérylène & $102 \%$ & $7 \%$ \\
\hline
\end{tabular}

Le protocole optimisé est décrit dans le tableau 6. Ce protocole permet d'obtenir des rendements de l'ordre de $70 \%$ à $100 \%$ pour tous les composés (tab. 7). La méthode peut être utilisée en mode manuel, sur une rampe d'extraction sous vide pour SPE disque, ou en mode automatique, avec un système d'extraction automatique (exemple SPE-DEX ${ }^{\circledR}$ 4790).

\subsection{Comparaison de deux approches pour l'extraction des HAP dans l'échantillon d'eau totale}

La DCE a défini les $N Q E$ dans des eaux totales ce qui inclut l'analyse de la phase dissoute et de la phase particulaire. Or les HAP (plus particulièrement les HAP à cinq et six cycles aromatiques) s'absorbent fortement aux particules. Une attention particulière est alors nécessaire

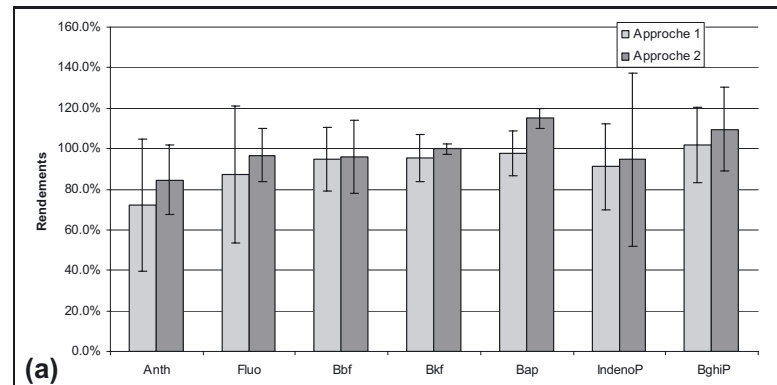

(a)

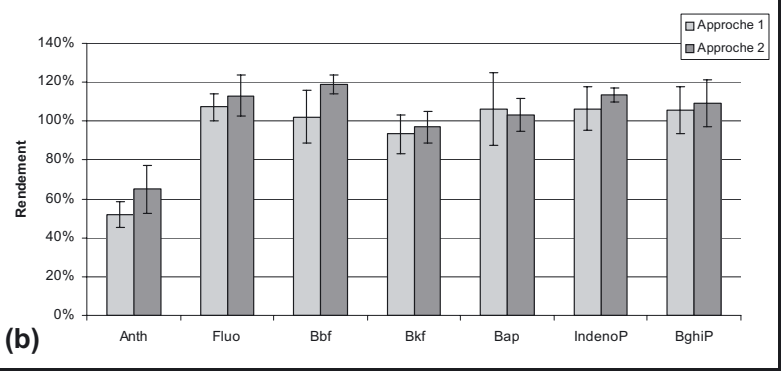

Fig. 2. - Rendements des deux approches pour l'extraction des HAP dans des échantillons d'eau contenant $5 \mathrm{mg} \cdot \mathrm{L}^{-1}$ d'acide humique et (a) $160 \mathrm{mg} \cdot \mathrm{L}^{-1}$ de MES $(n=3)$, (b) $400 \mathrm{mg} \cdot \mathrm{L}^{-1} \mathrm{de}$ $\operatorname{MES}(n=3)$.

pour assurer l'extraction complète des composés adsorbés [4]. Deux approches sont alors envisageables :

- deux extraction distinctes après séparation des particules en suspension et de l'échantillon filtré (approche 1) : recombinaison des extraits issus de l'extraction de la fraction dissoute par SPE disque et de l'extraction par PLE de la fraction particulaire;

- une seule extraction de l'eau totale par SPE disque (approche 2).

Ces deux approches ont été comparées pour déterminer celle qui présente les meilleures performances.

Les échantillons synthétiques analysés sont composés de $1 \mathrm{~L}$ d'eau minérale dopée avec $5 \mathrm{mg} \cdot \mathrm{L}^{-1} \mathrm{~d}$ 'acides humiques et deux quantités différentes de sédiments fournis par l'IRMM : $160 \mathrm{mg} \cdot \mathrm{L}^{-1}$ du MES HAP IRMM et $400 \mathrm{mg} \cdot \mathrm{L}^{-1}$ de MES Blanc IRMM (tab. 2). Chaque échantillon a été analysé en triplicat (fig. 2a et 2b).

Il n'y a pas de différence significative entre les deux approches. Cependant l'approche 1 nécessite un plus grand nombre d'étapes intermédiaires : filtration de l'échantillon, récupération du résidu du filtre qui sera 
Tableau 8

$L Q$ pour l'analyse des HAP dans l'eau totale par SPE disque-DI-CG/SM.

\begin{tabular}{|c|c|c|}
\hline Composé & $L Q\left(\mathbf{n g} \cdot \mathbf{L}^{-1}\right)$ & $\begin{array}{l}L Q \text { requise par } \\
\text { la } D C E\left(n g \cdot L^{-1}\right)\end{array}$ \\
\hline Anthracène & 2,4 & 30 \\
\hline Fluoranthène & 1,0 & 30 \\
\hline Benzo(b)fluoranthène & \multirow{2}{*}{0,6} & \multirow{2}{*}{9} \\
\hline Benzo(k)fluoranthène & & \\
\hline Benzo(a)pyrène & 0,3 & 15 \\
\hline Indeno(123-cd)pyrène & \multirow{2}{*}{1,7} & \multirow{2}{*}{0,6} \\
\hline Benzo(ghi)pérylène & & \\
\hline
\end{tabular}

extrait par solvant chaud pressurisé et extraction du filtrat par SPE disque. L'approche 2, étant la plus aisée et la plus rapide à mettre en œuvre, a été retenue.

\subsection{Validation de la méthode}

La méthode d'analyse des HAP dans l'eau totale par extraction SPE disque suivie d'une analyse par DI-CG/SM a été évaluée en termes de limite de quantification et de niveaux d'incertitude dans deux instituts. Afin de s'affranchir des différences liées aux pratiques des différents laboratoires nationaux de métrologie impliqués, les essais ont été réalisés par les collaborateurs en suivant un protocole commun.

Des échantillons synthétiques contenant $1 \mathrm{~L}$ d'eau minérale, $5 \mathrm{mg} \cdot \mathrm{L}^{-1} \mathrm{~d}$ 'acides humiques et deux quantités variables de matières en suspension (MES, simulées par des sédiments contenant naturellement des HAP (tab. 2)) ont été analysés en triplicat par le BAM et le LNE en suivant le même protocole opératoire.

\subsubsection{Estimation des limites de quantification (LQ)}

Une des exigences de performances de la DCE est d'atteindre une $L Q$ inférieure ou égale à $30 \%$ de la $N Q E$ [3]. C'est pourquoi des analyses ont été réalisées à $30 \%$ de la $N Q E$, à la $N Q E$ et également à trois fois la $N Q E$ pour couvrir l'ensemble du domaine.

Les $L Q$ ont été estimées en fonction du rapport signal sur bruit. Des échantillons d'eau minérale ont été dopés avec des acides humiques $\left(5 \mathrm{mg} \cdot \mathrm{L}^{-1}\right)$ et des sédiments $\left(100 \mathrm{mg} \cdot \mathrm{L}^{-1}\right)$ contenant de faibles concentrations de HAP. La $L Q$ a été déterminée selon l'équation : $\frac{S}{N}=\frac{2 H}{h_{N}}$ [27] avec $S / N$ le rapport signal sur bruit, $H$, la hauteur du pic chromatographique du HAP ciblé et $h_{N}$, hauteur du bruit au plus près du pic.

Les valeurs de $L Q$ obtenues (tab. 8) respectent les exigences de la DCE pour la majorité des composés, sauf pour l'indéno(123-cd)pyrène et le benzo(ghi)pérylène pour lesquels les valeurs obtenues sont supérieures aux $L Q$ exigées par la DCE mais comparables aux $N Q E$ (1,7 ng. $\mathrm{L}^{-1}$ obtenu par la méthode contre $0,6 \mathrm{ng} \cdot \mathrm{L}^{-1} \mathrm{de}-$ mandé par la DCE). Ces deux composés font partie des HAP les plus lourds (avec six cycles aromatiques) et sortent de la colonne à des temps de rétention très élevés en chromatographie gazeuse (de l'ordre de $30 \mathrm{~min}$ ). La sensibilité de l'appareil pour ces composés est donc faible et il est difficile de descendre à des concentrations de l'ordre de $1 \mathrm{pg} \cdot \mathrm{L}^{-1}$ pour ces composés. Plusieurs solutions sont possibles :

- augmenter le facteur de re-concentration en travaillant sur des volumes plus élevés d'échantillon au risque d'augmenter le temps d'extraction de l'échantillon, la difficulté de manutention de volume important d'eau et le risque de colmatage des disques SPE;

- utiliser un injecteur large volume permettant d'injecter des volumes plus élevés d'échantillon mais également des interférents.

\subsubsection{Estimation des incertitudes au niveau de la NQE}

Les incertitudes de mesure de la méthode ont été évaluées en suivant le GUM (Guide de l'expression des incertitudes de mesures) [28]. Cette méthode comporte trois étapes :

- les deux premières étapes consistent à définir le modèle et à évaluer la contribution de chaque paramètre dans le budget global de l'incertitude. La détermination de la concentration des HAP dans l'eau en $\mu \mathrm{g} \cdot \mathrm{L}^{-1}$ peut être décrite par l'équation (1) :

$$
\begin{aligned}
C_{\mathrm{M} / \text { éch }} & =\frac{C_{\mathrm{M}^{*} / \text { éch }} \times m_{\mathrm{M}^{*} / \text { éch }}}{m_{\text {éch }}} \times Q_{\text {éch }} \times f_{\mathrm{Q}_{\text {étal }}}+f_{\mathrm{F}} \\
\text { avec }: Q_{\text {éch }} & =a \times R_{\text {éch }}+b \\
C_{\mathrm{M}^{*} / \text { éch }} & =f_{\text {dil }^{*}} \times C_{\text {réf }^{*}}
\end{aligned}
$$

avec :

$C_{\mathrm{M} / \text { éch }}$ : concentration de la molécule $\mathrm{M}$ dans l'échantillon;

$C_{\mathrm{M}^{*} / \text { éch }}$ : concentration de la molécule $\mathrm{M}^{*}$ dans la solution introduite dans l'échantillon;

$m_{\mathbf{M}^{*} / \text { éch }}$ : masse de solution de $\mathbf{M}^{*}$ ajoutée à l'échantillon;

$m_{\text {éch }}$ masse prélevée d'échantillon;

$Q_{\text {éch }}$ : rapport de quantité de matière dans l'échantillon;

$f_{\mathrm{Q}_{\text {étal }}}$ : facteur de correction lié à l'incertitude de la concentration des solutions étalons ;

$f_{\mathrm{F}}$ : facteur de correction lié à la fidélité intermédiaire;

$R_{\text {éch }}$ : rapport de l'aire du signal correspondant à A sur l'aire du signal correspondant à $\mathrm{B}$ dans l'échantillon;

$f_{\text {dil }^{*}}$ : facteur de dilution qui tient compte de la préparation de la solution de $\mathbf{M}^{*}$ introduite dans l'échantillon à partir d'une solution mère de molécule marquée ;

$C_{\text {réf* }}$ : concentration d'une solution mère de molécule marquée. 
Tableau 9

Incertitudes de mesure pour l'analyse des HAP dans l'eau totale contenant deux niveaux de MES par SPE disque-DI- CG/SM (les cases en gris sont les concentrations les plus proches des valeurs $N Q E$ ).

\begin{tabular}{|l|c|c|c|c|}
\cline { 2 - 5 } \multicolumn{1}{c|}{} & \multicolumn{2}{c|}{$\begin{array}{c}\text { 160 mg MES HAP } \\
\text { IRMM }\end{array}$} & \multicolumn{2}{c|}{$\begin{array}{c}\text { M00 mg MES blanc } \\
\text { IRMM }\end{array}$} \\
\hline & $\begin{array}{c}\text { Concentration } \\
\left(\mu \mathrm{g} \cdot \mathrm{L}^{-1}\right)\end{array}$ & $\begin{array}{c}\text { Incertitude } \\
(k=2)(\%)\end{array}$ & $\begin{array}{c}\text { Concentration } \\
\left(\mu \mathrm{g} \cdot \mathrm{L}^{-1}\right)\end{array}$ & $\begin{array}{c}\text { Incertitude } \\
(k=2)(\%)\end{array}$ \\
\hline Ant & 0,086 & 23 & 0,003 & 11 \\
\hline Fluo & 1,489 & 8 & 0,024 & 9 \\
\hline BbF & 0,475 & 5 & 0,019 & 13 \\
\hline BkF & 0,262 & 7 & 0,009 & 21 \\
\hline BaP & 0,347 & 8 & 0,017 & 11 \\
\hline IndenoP & 0,463 & 8 & 0,020 & 16 \\
\hline BghiP & 0,470 & 5 & 0,023 & 26 \\
\hline
\end{tabular}

- la troisième étape est la détermination de l'incertitude composée en utilisant la loi de propagation de l'incertitude (approche «bottom-up» du GUM [21]) dont la formule générale est décrite par l'équation (2):

$$
\begin{aligned}
& u_{c}(y)= \\
& \sqrt{\sum_{i=1}^{N}\left(\frac{\partial f}{\partial x_{i}}\right)^{2} \times u^{2}\left(x_{i}\right)+2 \sum_{i=1}^{N-1} \sum_{j=i+1}^{N}\left(\frac{\partial f}{\partial x_{i}}\right)\left(\frac{\partial f}{\partial x_{j}}\right) u\left(x_{i}, x_{j}\right)}
\end{aligned}
$$

avec :

$u_{c}(y)$ : incertitude standard combinée de $y$;

$f: \quad$ modèle mathématique qui décrit $y$;

$x_{i}$ : $\quad$ paramètre de l'équation $y$;

$u\left(x_{i}, x_{j}\right)$ : covariances de $x_{i}$ et $x_{j}$.

- enfin la dernière étape consiste en l'expression de l'incertitude élargie «avec pour objectif de fournir un intervalle de $y-U$ à $y+U$ dont on peut s'attendre à ce qu'il comprenne une fraction élevée de la distribution des valeurs qui pourraient être attribuées raisonnablement au mesurande $Y »[28]$. Par convention, l'incertitude élargie est obtenue en multipliant l'incertitude type composée par un facteur d'élargissement $(k)$ de 2 pour assurer un intervalle ayant un niveau de confiance de $95 \%$ pour une distribution normale de la population.

L'incertitude de mesure au niveau de la $N Q E$ satisfait l'exigence de la DCE (tab. 9), c'est-à-dire est inférieure ou égale à $50 \%(k=2)$.

La composante majeure de l'incertitude est la composante de fidélité (fig. 3). Cette observation est explicable par le fait que les essais de validation de la méthode SPE disque-DI-CG/SM ont été réalisés dans deux instituts différents équipés de deux systèmes d'extraction SPE différents : LNE, système SPE disque automatique et au BAM, système SPE disque manuel. Les résultats de ces deux instituts ont été combinés pour estimer l'incertitude de mesure finale.

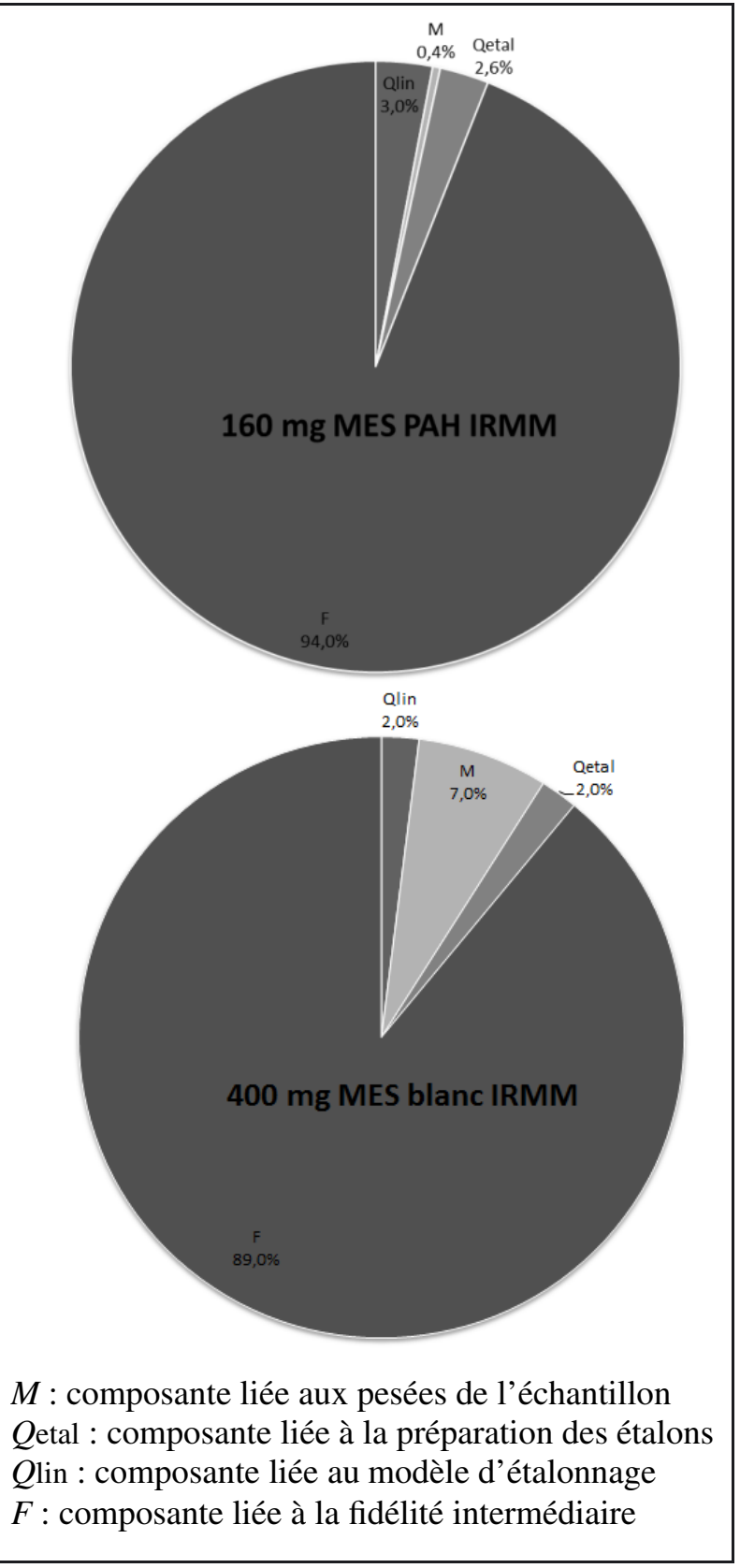

Fig. 3. - Composantes de l'incertitude de mesure pour l'analyse des HAP dans l'eau totale. 
Tableau 10

NQE établie en 2013 suite à la révision de la DCE.

\begin{tabular}{|c|c|}
\hline Composé HAP & $\begin{array}{c}\text { NQE 2013 } \\
\left(\boldsymbol{\mu g} \cdot \mathbf{L}^{-\mathbf{1}}\right)\end{array}$ \\
\hline Ant & 0,1 \\
\hline Fluo & 0,0063 \\
\hline BbF & ${ }^{(1)}$ \\
\hline BkF & ${ }^{(1)}$ \\
\hline BaP & $1,7 \times 10^{-4}$ \\
\hline IndenoP & ${ }^{(1)}$ \\
\hline BghiP & ${ }^{(1)}$ \\
\hline
\end{tabular}

(1) La $N Q E$ dans l'eau se rapporte à la concentration de benzo(a)pyrene, sur la toxicité duquel elle est fondée. Le benzo(a)pyrène peut être considéré comme un marqueur des autres HAP et, donc, seul le benzo(a)pyrène doit faire l'objet d'une surveillance aux fins de la comparaison avec la $N Q E$ dans l'eau correspondante.

\section{Conclusion}

Un des objectifs du projet européen ENV08 était de fournir une méthode de référence primaire pour l'analyse de sept HAP de la liste de substances prioritaires de la DCE dans l'eau conformément aux exigences réglementaires concernant les valeurs des concentrations et des incertitudes.

L'analyse des HAP dans l'eau totale (fraction dissoute et fraction particulaire comme exigée par la DCE) par extraction SPE disque suivie d'une analyse par DI$\mathrm{CG} / \mathrm{SM}$ a été validée pour cinq HAP pour répondre aux exigences de la DCE en termes de $L Q$ (inférieure ou égale à $30 \%$ des $N Q E$ ) et de niveau d'incertitudes (inférieure ou égale à $50 \%$ au niveau de la $N Q E$ ) : anthracène, fluoranthène, benzo(b)fluoranthène, benzo $(\mathrm{k})$ fluoranthène et benzo(a)pyrène.

Pour deux des HAP (indéno(123-cd)pyrène et benzo(ghi)pérylène), les $L Q$ n'ont pas été atteintes. Ces deux composés ont un temps de rétention élevé, et le signal obtenu en $\mathrm{CG} / \mathrm{SM}$ est bien inférieur à celui d'autres composés malgré l'étape de pré-concentration de l'échantillon et l'utilisation de grand volume d'injection. Cependant, comme l'indique la directive QA/QC [3] « en l'absence de norme de qualité environnementale appropriée pour un paramètre donné ou en l'absence de méthode d'analyse répondant aux critères de performance minimaux visés au paragraphe 1, les États membres veillent à ce que la surveillance soit effectuée à l'aide des meilleures techniques disponibles n'entraînant pas de coûts excessifs », et, à ce jour, la méthode fournie dans le projet ENV08 répond à ces critères.

La nouvelle DCE, Directive 2013/39 / UE [12], établit de nouvelles valeurs $N Q E$ pour les HAP (tab. 10). La méthode validée dans le projet EMRP ENV08 ne pourra pas répondre aux nouvelles exigences en termes de niveau de concentration. Une alternative, qui pourrait donner lieu à une prochaine étude, serait d'analyser les HAP dans le biote avec une $N Q E$ de $5 \mathrm{ng} / \mathrm{g}$, limite pouvant être atteinte.

\section{Remerciements}

La recherche menant à ces résultats a été réalisée dans le cadre du projet de recherche de l'EMRP ENV08 «Traçabilité des mesures pour la surveillance des polluants critiques de la Directive Cadre Européenne sur l'Eau (2000/60/CE) ». L'EMRP est un programme de recherche d'Euramet financé conjointement par les pays participants à l'EMRP et par l’Union Européenne.

\section{Références}

[1] Directive 2000/60/EC du parlement européen et du conseil du 23 octobre 2000 établissant un cadre pour une politique communautaire dans le domaine de l'eau.

[2] Directive 2008/105/CE du parlement européen et du conseil du 16 décembre 2008 établissant des normes de qualité environnementale dans le domaine de l'eau, modifiant et abrogeant les directives du Conseil 82/176/CEE, 83/513/CEE, 84/156/CEE, 84/491/CEE, 86/280/CEE et modifiant la directive 2000/60/CE.

[3] Directive 2009/90/EC de la commission du 31 juillet 2009 établissant, conformément à la directive 2000/60/CE du Parlement européen et du Conseil, des spécifications techniques pour l'analyse chimique et la surveillance de l'état des eaux.

[4] Lepom P., Brown B., Hanke G., Loos R., QUEVAUVILLIER P. et Wollgast J., "Needs for reliable analytical methods for monitoring chemical pollutants in surface water under the European Water Framework Directive", J. Chromatography A, 1216, 2009, 302-315.

[5] Coquery M., Morin A., Bécue A. et Lepot B., "Priority substances of the European Water Directive: analytical challenges in monitoring water quality", Trends Anal. Chem., 2005, 117-127.

[6] ISO 17993, 2002, "Water Quality - Determination of 15 polycyclic aromatic hydrocarbons $(\mathrm{PAH})$ in water by HPLC with fluorescence detection after liquid-liquid extraction".

[7] ISO/DIS 28540, 2011, "Water Quality - Determination of 16 polycyclic aromatic hydrocarbons (PAH) in water Method using gas chromatography with mass spectrometric detection (GC-MS)".

[8] EPA Method 525, "Determination of organic compounds in drinking water by liquid-solid extraction and capillary column gas chromatography".

[9] Erger C., BalsaA P., Werres F. et Schmidt T.C., "Multi-component trace analysis of organic xenobiotics in surface water containing suspended particular matter by solid phase extraction/gas chromatography-mass spectrometry", J. Chromatography A, 1249, 2012, 181-189.

[10] Ademollo N., Patrolecco L., Polesello S., VAlSECChI S., Wollgast J., Mariani G. et HaNKe G., "The analytical problem of measuring total concentration of organic pollutants in whole water", Trends Anal. Chem., 2012, 71-81. 
[11] Werres F., BalsaA P. et Schmidt T.C., "Total concentration analysis of polycyclic aromatic hydrocarbons in aqueous samples with high suspended particulate matter content", J. Chromatography A, 1216, 2009, 2235-2240.

[12] THURMAN E.M., "Developments in biogeochemistry: Organic geochemistry of natural waters", Ed. Nijhoff, Dr. W. Junk Publishers, Dordrecht, 1985.

[13] LI N. et LEE H.K., "Solid-phase extraction of polycyclic aromatic hydrocarbons in surface water - Negative effect of humic acid", J. Chromatography A, 921, 2001, 255-263.

[14] JEANNEAU L., FAURE P. et JARdÉ E., "Influence of natural organic matter on the solid-phase extraction of organic micropollutants - Application to the water-extract from highly contaminated river sediment", J. Chromatography A, 2007, 1-9.

[15] Bercaru O., Ulberth F., EmONS H. et VANDECASTEELE C., "Accurate quantification of PAHs in water in the presence of dissolved humic acids using isotope dilution mass spectrometry", Anal. Bioanal. Chem., 384, 2006, 1207-1213.

[16] Peignaux M., Cabillic J., Lalere B. et Macé T., «Développement de matériaux de référence pour les HAP », LCSQA, Rapport, 2008.

[17] Wolska L., GdANiEC-Pietryka M., KonieczKa P. et NAMIESNIK J., "Problems of PAH quantification by GCMS method using isotope-labelled standards", Talanta, 2009, 730-735.

[18] ITOH N., MasahiKo N., AOYAGi Y. et YARITA T., "Effect of residues remaining in the injection liner of a chromatograph on the quantification of polycyclic aromatic hydrocarbons by isotope dilution mass spectrometry using deuterium-labeled internal standards", J. Chromatography A, 1134, 2006, 246-252.

[19] Van de Kreek J., De la Calle B., Held H., Bercaru O., Ricci M., SHEGUNOVA P. et TAYLOR P., "IMEP-23 the eight WFD PAHs in water in presence of humic acids", Trends Anal. Chem., 2010, 928-937.

Article reçu le 2 février 2015, version révisée reçue le $1^{\text {er }}$ octobre 2015.
[20] Note d'application $n^{\circ} \mathrm{TN}-0042$, "Improved recoveries of Polycyclic Aromatic Hydrocarbons (PAHs) as defined in EPA 550.1 and simultaneous removal of Humic Acids from water using Strata ${ }^{\circledR}$ PAH", Phenomenex.

[21] PR NF EN 16691, «Qualité de l'eau - Dosage des hydrocarbures aromatiques polycycliques (HAP) dans des échantillons d'eau totale par extraction liquidesolide avec couplage chromatographie en phase gazeusespectrométrie de masse (CG-SM) ».

[22] MACÉ R.M. et BorRULl F., "Solid-phase extraction of polycyclic aromatic compounds", J. Chromatography A, 885, 2000, 273-290.

[23] Barrek S., Cren-Olivé C., Wiest L., Baudot R., ARNAUdGUILHEM C. et GRENIER-LOUSTALOT M.-F., "Multi-residue analysis and ultra-trace quantification of 36 priority substances from the European Water Framework Directive by GC-MS and LC-FLD-MS/MS in surface waters", Talanta, 79, 2009, 712-722.

[24] Note d'application $n^{\circ} 301260$, "PAH from water containing Humic Acids", Macherey-Nagel.

[25] Note d'application $\mathrm{n}^{\circ}$ 301250, "PAH from water, Macherey-Nagel".

[26] Note d'application $\mathrm{n}^{\circ}$ AN008-091208, "Comparison of analyte recoveries using Atlantic ${ }^{\mathrm{TM}} \mathrm{C} 18$ and JT Baker Speedisk ${ }^{\circledR}$ Octadecyl (C18) for EPA 525.2”, Horizon technology.

[27] DIN 32645:2008, "Chemical analysis - Decision limit, detection limit and determination limit under repeatability conditions - Terms, methods, evaluation".

[28] ISO/IEC Guide 98:2008, "Guide to the expression of uncertainty in measurement", 2008.

[29] "Determination of polycyclic aromatic hydrocarbons in drinking water by liquid liquid extraction and HPLC with coupled ultraviolet and fluorescence detection", Environmental Monitoring Systems Laboratory Office Of Research And Development U.S. Environmental Protection Agency Cincinnati, Ohio, J.W. Hodgeson, Method 550, juillet 1990.

[30] NF EN ISO 17993, juillet 2004, «Dosage de 15 hydrocarbures aromatiques polycycliques (HAP) dans l'eau par HPLC avec détection par fluorescence après extraction liquide-liquide ». 